\title{
Viable System Model approach for holonic product-driven manufacturing systems
}

\author{
Carlos Herrera $^{\star}$, Sana Belmokhtar Berraf, and André Thomas \\ Center for Automatic Control of Nancy, Nancy Université, CNRS UMR 7039 \\ Faculté des sciences - BP 23954506 Vandoeuvre les Nancy - France \\ \{carlos.herrera, sana.belmokhtar, andre.thomas\}@cran.uhp-nancy.fr \\ http://www.cran.uhp-nancy.fr
}

\begin{abstract}
The paper presents a generic framework for a Product Driven Control Systems (PDCS) dealing with production planning and control. The framework is based on Viable System Model (VSM) which is introduced for intelligent manufacturing systems. Based on suitable properties as autonomy, self-organization and adaptability, VSM allows to model and consider these properties for PDCS. An application for a Manufacturing Planning and Control System (MPCS) is proposed. Different points of view are also presented based on this application which are showed and explained in the sens of VSM principles. Finally, a discussion is presented dealing with the main issues of the proposed approach.
\end{abstract}

Keywords: Product-driven systems, intelligent manufacturing systems, viable system model, manufacturing planning and control

\section{Introduction}

The economic context including globalization and free competition triggers quick evolutions of several technologies available for the industry and users. That pushes firms to be more reactive, more innovative, more agile.

In this context, Intelligent Manufacturing Systems (IMS) (arisen in the earlies 90's), aim to improve the conventional Manufacturing Planning and Control Systems (MPCS) in terms of flexibility, reconfiguration and scalability, among others. In this class of systems, Product-Driven Control Systems (PDCS) [11], based on intelligent products [12], change the vision of products from a passive state into active actors in the decision process concerning itself [13]. Nevertheless, one of the major limitation of these kind of systems is the absence of specific frameworks to guide the process of analysis, design and implementation.

For that purpose, in this paper, we propose a general framework to model product-driven control systems applied to MPCS. The proposed framework is designed to be used in a wide range of production or services product-driven systems. This leads us to a generic concept which provide sufficient theoretical concepts and implementation guidelines to manage the inherent complexity of this kind of systems.

\footnotetext{
* Corresponding author
} 
Our work has been motivated by the design of intelligent products as living beings, focusing our research in theories and models concerning the structure and organization of these living machines [10]. The objective is to use such models to design manufacturing systems that can show properties like autonomy, selforganization and adaptability. These properties can be summarized as viability commonly present in living beings. The presence of such properties has lead to the conceptual framework present in the Viable System Model [3,4]. The choice of VSM has been made since this framework is suitable to model these properties.

Main property in VSM is recursion, that allows to manage complexity and find a compromise between control and autonomy to implement principles as heterarchy and cooperation [6]. These are central elements of product-driven control systems and in a more general way a key objective of IMS. Manage complexity allows to consider each system as a whole preserving the emergent properties which can only be understood using a holistic vision, in contrast to reductionist ones.

VSM since its origin in the earlies 70's as result of the research of Stafford Beer, has been object of a constant validation with several applications until nowadays. The pertinence of this model can be justified regarding to the recent applications concerning domains like material management $[8,15]$, autonomous distributed systems [14], autonomous software [7,9] or autonomous communities of agents [17].

Our contribution deals with the use of VSM applied to PDCS as an integrated modeling framework. The most important paradigms arisen from IMS are biological [18], fractal [20] and holonic manufacturing systems [19]. The biological paradigm and properties like synthesis has been essential parts of biological systems, recursion is the basis property of fractal system and the difficulty to separate parts from the whole is a main concept in holonic systems. Our intention is to show that VSM can be used like a consensual framework for all these research threads.

This paper is organized as follows: section 2 presents a general description of the main concepts involved in VSM, and presents the approach focusing on the elementary object of the proposed structure, that is the intelligent product. Section 3 describes an application of the proposed approach for a Manufacturing Planning and Control System. In section 4 a discussion of the main findings is presented. Finally, section 5 presents the conclusion.

\section{$2 \quad$ VSM for holonic product-driven manufacturing systems}

The origins of VSM arise from the work of Beer applied to the steel industry in the fifties. This research can be placed in the line of works of Norbert Wiener, Warren McCulloch and Ross Ashby [5]. The main objective of the model was to identify and to explain how systems are viable, in other words, how it is possible that some systems can maintain an independent existence. 
Although VSM is a general model for the study of any viable system, the most concerned application area has been human activity organizations, i.e., corporations, firms or governments [2]. In this domain, VSM changes the view of the traditional management model based on command and control, in which a control system is designed as a pyramid and such decisions are disaggregated in a top-down manner at different structural levels. The main difference, inspired by the biological organization, consists in mapping this hierarchy into a structural recursion.

The premise of this change of perspective was inspired from the living beings composition (cells, organs, systems, etc.). Indeed, they have properties of autonomy, self-organization and self-regulation, allowing them to have an independent existence. The differentiation of their functions and the relationships between these elementary components produce more complex systems, without that subsystems essential properties would be lost. However, one of the most important properties of a viable system is their intrinsic recursion. In fact, any viable system contains and is contained by another viable system. Every subsystem maintains its autonomy towards its environment, but it also contributes to generate the viable system in which it is included. In that way, a viable system and its different subsystems have the same structural requirements. A viable system supports its objectives thanks to an overall cohesion and adapts itself by the autonomy of its subsystems.

VSM was developed looking for invariances in organic systems. These invariances allow to define a homomorphism of their functions, organization and structure. Beer defines five elementary functions that any viable system must have: implementation, coordination, control, intelligence and policy. In the following are defined more in details each one of these functions:

Implementation : this function refers to primary activities that materialize the identity of the system. These activities are identified after an analysis of what the system does, then directly related to the system transformation and environment interaction.

Coordination : this function corresponds to the coordination among primary activities. In a viable system, this coordination is not necessarily accomplished in a top-down manner like in a hierarchical management system. Primary activities can be coordinated in a centralized or distributed way thanks to cooperation and information exchanges.

Control : control refers to the function which regulates and ensures the selforganization of the system. This is due to coordination and monitoring functions. Monitoring function (or sporadic audit function) allows at the same time to evaluate the actions of primary activities and to hold the coherence of the global activity (all primary activities). Control function is the function that defines the control/autonomy degree of primary activities.

Intelligence : the intelligence function is responsible for system adaptability. To achieve this adaptation, the intelligence function must be capable of treating the information which comes from the environment, with the objective to anticipate perturbations. 
Policy : the policy function keeps the system objectives at its level.

As it was expressed by Ashby [1] in the law of requisite variety a controller will be effective only if it is capable of attaining at least the same number of states as those which it wants to control. The variety of a system is defined as all possible states of a system and it can be used as a complexity measure.

"A controller has requisite variety - that is, has the capacity to maintain the outcomes of a situation within a target set of desirable states - if and only if it has the capacity to produce responses to all those disturbances that are likely to take the outcomes out of the target set".

To achieve that, the viable system tries to reduce the variety coming from the environment and to amplify the variety of its control function to reach a balance (homeostasis). Figure 1 shows an example of a viable system which is decomposed into three subsystems. The recursion property of VSM allows continuing this process until the required level of detail. In the example presented in figure 1, it is possible to break down each of the three viable systems in new viable sub-systems. Also, the whole system can be considered as a subsystem of another viable system at an upper recursive level. Notice that all systems have the same structure.

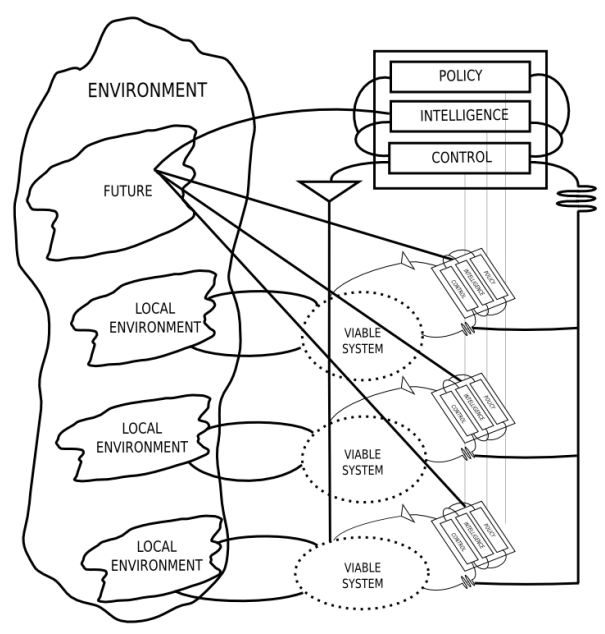

Fig. 1. A Viable System Model

A conventional recursive decomposition of a production system, will decompose the system, for example, on the shop-floor, production cells, production lines, production modules and machines. In such case, machines would be the elementary (atomic) entities of the system at the lowest recursion level.

In contrast with this conventional interpretation, we consider the product to be the elementary decision-making entity responsible for driving work tasks. Therefore, it is considered as the basic element of the production process and it 
is designed as a viable system. Others system entities such as machines, conveyors, automated guided vehicles, team workers, etc., are then considered in the environment. These entities interact with the product only in terms of events leading to lack of availability, disruptions, constraints, etc. Figure 2 shows the intelligent product structure which is proposed.

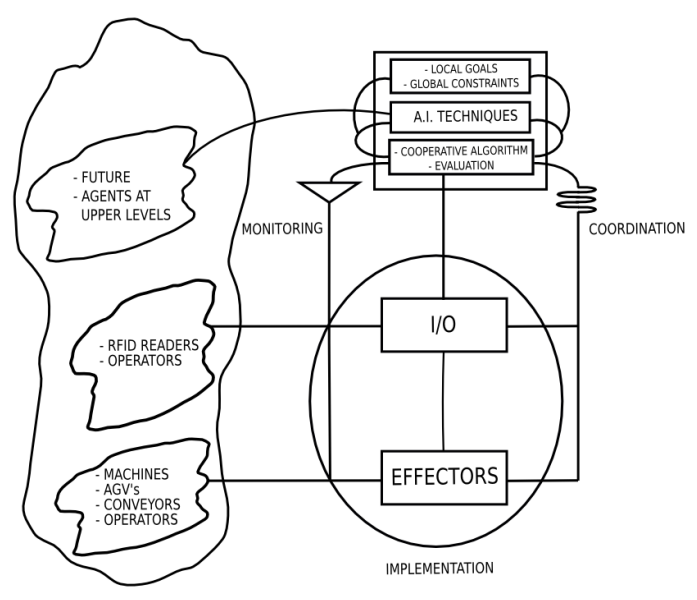

Fig. 2. An intelligent product modeled using VSM

As it has been said in the introduction, the intelligent product is defined as an entity able of interacting and communicating with its environment. When this physical entity transits on the shop-floor, it will be able to recover information concerning both its own state and the system state. Then, focusing on the product, all the production planning system can be structured from the information recovered by this intelligent product. Also, decision-making problems are addressed to their corresponding recursion level.

The primary activities of the intelligent product are data collection and interaction functions. The data collection activity is designed as the action which allows the product to get information from other products and to give information to them. On the other hand, interaction functions allow products to interact with their environment.

\section{Application to MPC systems}

Manufacturing Planning and Control Systems contain five main functions which can be described as : Strategic Planning, Sales and Operations Planning (S\& OP), Master Production Planning (MPS), scheduling and execution. Each of these functions corresponds to a level in the decision making process regarding 
to different horizons going from a longer to shorter one. In practice, these decisions are taken using a rolling horizon to take into account the frequent changes that occur in the data (demand, capacity, etc.). Thus, the strategic planning is revised once a year, the S\&OP is computed monthly, the MPS is get per week, and the schedules are performed daily or more frequently depending on disturbances. Each function deals with a corresponding aggregation level of products respectively families, finished products and items (components). In this context, one of the major issue is to adapt decisions at each level when disturbances (internal or external) happen. The frequent resulting modifications in the decision making process lead to the so-called nervousness system which deteriorates the system performance (productivity and efficiency). One should notice, that the shorter is the horizon the more frequent are the changes. Thus, the performance are more deteriorated at the lower level (scheduling level).

More precisely, our proposition is to provide a generic model based on VSM dealing with production planning considering both MPS level and scheduling (lot-streaming). The MPS allows to define quantities for finished products per periods on a specific horizon. This is usually performed with a capacitated lotsizing problem which is classically formulated with a mixed integer program. This model is implemented at the higher level in the figure 3. Generally, once lot sizes are determined, sub-lots have to be sequenced and dimensioned to be launched on the shop-floor (lot-streaming problem) [16]. This model is implemented at the level two from the bottom in the figure 3 . Thus, different objectives could be reached corresponding to each decision level (minimizing stocks and set up costs at the MPS and minimizing makespan at lot-streaming level). However, disturbances are frequent in this context, such an example could be found in the demand changes or the capacity reduction (machine failure). As a consequence of these disturbances, the computed predictive planning would be inadequate and should be adjusted regarding to the new conditions (data). Nevertheless, the combinatorial nature and the variability of the different parameters of the considered problems do not allow an on-line computation. The characteristic of self-adaptation is then present with an adjustment of machine capacity when a failure happen in a certain limit. Obviously, when the capacity comes down a certain threshold then it becomes impossible to find a solution, in this case the problem is forwarded to the upper level (a bottom-up process).

For external disturbances, the centralized system modify the threshold and the global objectives to adapt itself in a top-down manner by defining new targets for the bottom level. At the opposite to the bottom-up decision making process which has a cooperative characteristic, the adjustment process (top-down) has a coercive characteristic.

The figure 3 shows an example of four recursion levels. This system has been proposed with the hypothesis of using intelligent products mainly concerned with RFID technology to embed and communicate information.

The proposed model aim to face external and internal disturbances which could occur. This system is based on a holarchy of products regarding to VSM modeling. Each decision level corresponds to a certain recursion level. The dif- 
ferent levels of recursion are defined as agents communities which should adapt themselves to the decision making conditions. Moreover, they have the objective to collaborate to find a suitable solution. Figure 3 is subdivided into 4 parts (I,II,III et IV) in order to simplify the explanations. The two axes allow to represent different points of view where the horizontal axis distinguish the physical world from the virtual one. Whereas the vertical axis shows at the left side the design which is a representation of the system. At the right side, the implementation deals with both the knowledge managements and decision making systems.

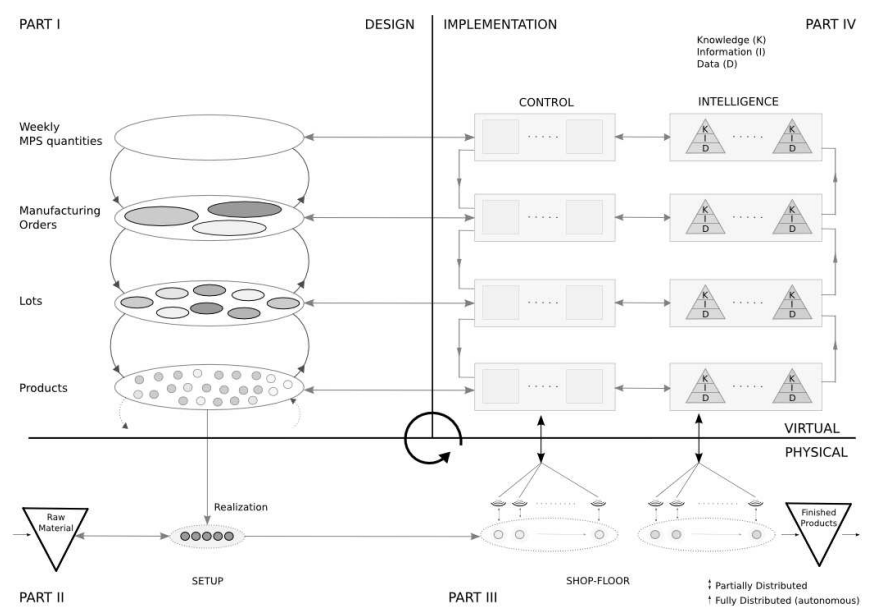

Fig. 3. VSM based product-driven control system applied to MPCS

\section{Part I : Design/Virtual}

This part is relative to the centralized or cooperative system representation. The representation is decomposed into four recursive levels of product entities. At each level, the concerned entities (weekly production, manufacturing orders, lots and products) are modeled thanks to the basic object proposed in the figure 2. To take decision, a product or a group of products will have policies, intelligence and control functions, coordination and communication mechanisms.

The products being defined as agents with a specific control/autonomy level, three alternatives are possible. First, if the system is strictly distributed or heterarchic, agents communicate only in a cooperative way for decision-making process. Second, if the system is strictly centralized, decision are taken with methods (models) that collect information thanks to agents and aggregate it to propose centralized planning orders. Third, between these two opposite solutions, many combinations are able to be implemented adapting different degree of control/autonomy. 


\section{Part II : Design/Physical}

This part of the figure is relative to the product instantiation at the physical level. Each of the four decision-making levels of the previous part could generate plans for the corresponding level. According to the physical/virtual nature of the entities, products will be built as holons in this part. They will have specific functions to be able to interact with their environment. Each of the four recursive levels would be designed like holons in the same way. For example, the physical part of a manufacturing order holon would be the manufacturing order paper form or a kanban card would be the physical part corresponding to a lot.

\section{Part III : Implementation/Physical}

In this part product holons are able to make decisions according to events concerning their own evolution. Specific functions are embedded in these intelligent products leading them to react to events in an individual or collective way.

\section{Part IV : Implementation/Virtual}

This last part is relative to the treatment of information coming from the shopfloor and concerning disruptions and product actions, on one hand. On the other hand, decision making process is also handled in this part. In the same way, each recursive level corresponds to a decision making one which interacts with the corresponding knowledge level. This treatment could be defined as a information and knowledge model [21].

\section{Discussion}

An important concern of VSM is adaptability as it was described in the previous section, VSM was developed with the objective to understand the mechanisms which allow biological organisms to survive to changes in the environment. A viable system is designed to self-adaptation in a changing environment, by the implementation of the necessary mechanisms to support its viability. Indeed, it is not possible to identify a specific function which ensures adaptability, since the whole system is defined to fulfill this objective, thanks to its different elementary functions. This characteristic of adaptability is also one of the main objectives of the paradigms arising from IMS initiative.

One of the basic properties of the viable systems is scalability because these systems are structurally recursive. This notion of recursion has been mainly exploited by biological manufacturing systems, which make an analogy from biological organisms. Recursion has been also exploited by fractal manufacturing systems which show clear properties of modularity and auto-similarity. Autosimilarity in fractal systems is also included in the viable systems through its property of structural recursion. In that way, a viable system will keep the same structural requirements at any complexity level. These concepts have a direct 
relationship with re-usability, which is another objective of IMS systems. These concerns of autonomy, adaptability and scalability highlight the strong relationship between VSM and IMS objectives.

Considering the context of our study dealing with the Product Driven Control Systems which consider the product as an intelligent entity, the idea of the proposed VSM model takes the product as the elementary (primary) recursive component. The others recursive levels of our model are established according to the classical decomposition resulting from MPCS management which provides : products, lots, manufacturing orders and MPS weekly quantities. Note that the product is a generic term and could be extended to component or raw material. In such case, corresponding lower levels should be added.

\section{Conclusion}

In this paper, we have presented the main concepts involved in the VSM and how those can be applied to model product-driven control systems. It has been highlighted that VSM has nice properties to model intelligent manufacturing systems. We have presented an application of the proposed modeling framework to model a MPCS product-driven system. The main advantage of this application is to reach a coherence among the different decision levels giving a high degree of flexibility to the system. The proposed modeling approach applied to productdriven systems could be implemented in different industrial context.

Acknowledgments. The authors gratefully acknowledge the financial support of the CPER 2007-2013 Competitiveness Fibre Cluster (Structuration du Pôle Compétitivité Fibres Grand'Est), local (Conseil Général des Vosges), regional (Région Lorraine), national (DRRT and FNADT) and European (FEDER) funds.

\section{References}

1. Ashby, R.: An introduction to cybernetics. Chapman and Hall, London (1957)

2. Beer, S.: The liberty machine. Futures 3, 338-348 (1971)

3. Beer, S.: Brain of the firm: the Managerial cybernetics of organization. Allen Lane the Penguin Press, London (1972)

4. Beer, S.: The viable system model: Its provenance, development, methodology and pathology. Journal of the Operational Research Society 35, 7-25 (1984)

5. Espejo, R.: The viable system model a briefing about organisational structure. Website (2003), http://maine.gov/dhhs/btc/PDF/ViableSytemModel.pdf

6. Herrera, C., Thomas, A., Belmokhtar, S., Pannequin, R.: A viable system model for product-driven systems. In: IESM 2011. To appear : In proceeding of the International Conference on Industrial Engineering and Systems Management (IESM) (2011)

7. Herring, C., Kaplan, S.: Viable Systems: The Control Paradigm for Software Architecture Revisited. Australian Software Engineering Conference pp. 97-105 (2000) 
8. Herrmann, C., Bergmann, L., Halubek, P., Thiede, S.: Lean production system design from the perspective of the viable system model. In: Mitsuishi, M., Ueda, K., Kimura, F. (eds.) Manufacturing Systems and Technologies for the New Frontier, pp. 309-314 (2008)

9. Laws, A., Taleb-Bendiab, A., Wade, S.: Genetically modified software: Realizing viable autonomic agency. Innovative Concepts for Autonomic and Agent-Based Systems pp. 184-196 (2006)

10. Maturana, H., Varela, F.: Autopoiesis and cognition: The realization of the living. Reidel Publishing, The netherlands (1980)

11. McFarlane, D.: Product identity and its impact on discrete event observability. In: Proceedings of the European Control Conference (ECC) (2002)

12. McFarlane, D., Sarma, S., Chirn, J., Wong, C., Ashton, K.: The intelligent product in manufacturing control. Journal of EAIA 5464 (2002)

13. Morel, G., Valckenaers, P., Faure, J.M., Pereira, C.E., Diedrich, C.: Manufacturing plant control challenges and issues. Control Engineering Practice 15, 1321-1331 (2007)

14. Ribeiro-Justo, G., Saleh, A., Karran, T.: Intelligent reconfiguration of dynamic distributed components. Electron. Notes Theor. Comput. Sci. 180, 91-106 (2007)

15. Rosenkranz, C., Holten, R., Laumann, M.: Designing ic structures by variety engineering. In: SAC'08: Proceedings of the 2008 ACM symposium on Applied computing. pp. 518-523 (March 2008)

16. Sarin, S., Jaiprakash, P.: Flow Shop Lot Streaming. Springer, New York (2007)

17. Truszkowski, W., Karlin, J.: A cybernetic approach to the modeling of agent communities. In: CIA '00: Proceedings of the 4th International Workshop on Cooperative Information Agents IV, The Future of Information Agents in Cyberspace. pp. 166-178 (July 2000)

18. Ueda, K., Vaario, J., Ohkura, K.: Modelling of biological manufacturing systems for dynamic reconfiguration. CIRP Annals-Manufacturing Technology 46(1), 343-346 (1997)

19. Valckenaers, P., Van Brussel, H.: Holonic manufacturing execution systems. In: CIRP annals. General Assembly of CIRP No55, vol. 54, pp. 427-432 (2005)

20. Warnecke, H.: The Fractal Company (1993)

21. Zins, C.: Conceptual approaches for defining data, information, and knowledge: Research articles. J. Am. Soc. Inf. Sci. Technol. 58, 479-493 (February 2007) 